directly impacts one of 4 key strategic priorities of STFM, that of financial independence. The long-term sustainability of STFM will be to some degree dependent upon the largesse of STFM members who contribute annually to the Foundation, and who remember STFM in their estate plans. With fewer than $10 \%$ of STFM members being Foundation donors, there is a lot of potential for additional support of leadership programs, research and curriculum development, and special projects. There is also the need to build reserves for operations in times of a slow economy. We know we need to keep moving forward; we know where we are going. If, as this quote says, "You are successful the moment you start moving toward a worthwhile goal," then the STFM Foundation is well on its way.

Peter Coggan, MD, MSEd, STFM Foundation President; Joan Morrill, STFM Foundation Chief Development Officer

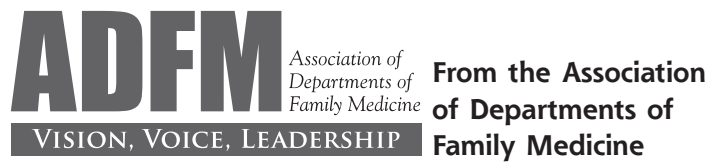

Ann Fam Med 2010;8:469-470. doi:10.1370/afm.1180.

\section{Family Medicine Clerkship, Tracks, and Faculty Support for Family Medicine Education in Departments of Family Medicine: An Update}

Departments of Family Medicine, while experiencing financial resource diminution, are simultaneously called upon to expand their primary care bases and teaching programs, while building and testing medical homes with a goal of advancing to fiscal independence. These rapid changes, while posing unique opportunities, have the potential to diminish and de-prioritize student education as practice transformation occurs.

The changing environment of academic and community clinical venues used for medical student education presents substantial challenges for family medicine educators. Evolving health care reform with greater emphasis on access, quality, outcomes, and appropriate reimbursement are some of the components forcing transformation of family medicine patient care environments to meet emerging primary care delivery challenges. These challenges are being addressed differently across diverse clinical venues potentially creating substantial inconsistency in the way concepts such as the Patient Centered Medical Home are implemented. These evolving primary care delivery settings will likely, create another level of resource inconsis- tency in clinical venues secured and used for family medicine clerkship teaching.

Adding to the challenges of ensuring consistent student teaching experiences in diverse clinical settings is the integration of the national Family Medicine Clerkship Curriculum. The Family Medicine Clerkship Curriculum provides the core portion of student clinical experiences and content that should be provided to students in all clerkship settings across the country, the "what" of family medicine student education. The Curriculum's national task force's decision to start with a small set of content expectations immersed in a patient-centered context may prove to be wise as a second project currently engaged by STFM seeks to determine the "how" as it relates to implementation of the Curriculum. The "how" will likely be complicated by the diversity of the clinical venues where medical students receive their core clinical family medicine training. These settings, which are usually distributed and/or decentralized, make it necessary for curriculum planners to create multiple paths to achieve similar curricular goals (tailoring clinical teaching to unique care delivery settings).

The ADFM's 2010 Quick Hitter Survey of 93 departments (66\% of all ADFM member departments) reveals the presence of a required family medicine Clerkship in $95 \%$ of responding departments with a wide range of resources available to support the clerkship experience. Eighty-four percent of these clerkships were in the 3 rd year with $32 \%$ reporting a 4 -week requirement, $45 \%$ a 6 -week requirement, and $11 \%$ an 8 -week requirement $(9.5 \%$ not mentioning length of time), and 5\% reporting clerkships with both 3rd- and 4th-year students. Multiple unique clerkship arrangements were reported such as 4 th-year requirements, longitudinal experiences, special tracts such as rural locations, special subject interests such as geriatrics, and various levels of integration with other primary care clerkship experiences. These different settings will demand unique implementation strategies to ensure appropriate integration of the new curriculum guidelines.

Efforts to develop consistent curricular core content for the family medicine clerkships will almost certainly require additional departmental investment to support implementation, maintenance, and evolution of these teaching experiences. Many family medicine clerkships are already highly dependent on volunteer teaching and the management of resources beyond the medical school. The dynamic practice transformation occurring in these clinical education sites must be evaluated to determine the implications of these changes on student education. If, especially in academic settings, planned practice transformation 
could consistently occur simultaneously with efforts to support the core content requirements of the family medicine clerkship, exportable teaching models for use in dispersed clinical settings may emerge.

The differences in the number of FTE's devoted to support medical student education varied greatly in the ADFM Quick Hitter Survey with the differences appearing not attributable to class size. The survey reveals responses concerning a variety of tracts for medical student learning to include rural, urban, and underserved, global/international health, research, and community/integrative health. Although these tracts may have unique importance for individual departments, the implication of these activities on the ability to deliver required family medicine teaching experiences consistent with national norms is unknown. Departments must carefully balance their practice transformational activities with the dynamic changes that come with evolving standardization of required family medicine teaching experiences occurring mostly in stand-alone family medicine clerkships as well as department-specific elective and selective activities.

This Annals commentary was prepared by the Chair and Vice Chair of the ADFM Medical Student Education Committee with review and comment by the ADFM Executive Committee Andrea Manyon, MD, Josepb Hobbs, MD, and the Association of Departments of Family Medicine

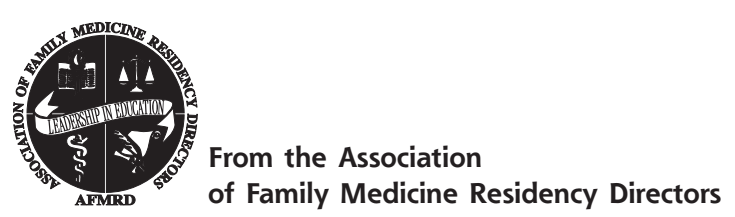

Ann Fam Med 2010;8:470. doi:10.1370/afm.1181.

\section{Residency "Dashboard": Family Medicine GME's Step Towards Transparency and Accountability?}

As we drive to work each day, we each see things unique to our locales, but one thing in common is that we all look at our vehicle's dashboard. We do this because it is one place that shows us information that affects each trip we make, unlike the weather outside, which may or may not have an impact on our commute. Some of us with clunkers may watch our dashboard more closely than those with a newer car. But even those with reliable vehicles need to know their speed, how much gas they have, or if the dreaded check engine light goes on!

How does one measure the quality of Family Medicine residency graduates or programs? Currently there are no uniform benchmarks of quality for either individual or residency comparisons. Some residencies have rankings and reputations based on certain criteria. Are those criteria valid for programs in all settings and do they predict any acceptable quality outcome indicators? Like it or not, our constituents (students, residents, patients) use a variety of non-standardized criteria to make judgments about our graduates and our programs. The AFMRD Board has made quality a pillar of its new 3-year strategic plan. One means by which we would like to advance this quality focus is in the potential use of a residency dashboard.

Going back to our car analogy, the concept of a dashboard for FM residency programs is much the same. The measures that would be reported and tracked would be those things common, yet vital, in terms of the health and quality of all programs. Measures such as board certification and match rates, scholarly output, accreditation status, community service, and resident turnover are just some of the possible dashboard indicators. Ultimately one would envision using more robust indicators such as program graduates' quality performance, community impact, and the RPS criteria met by each program. The long term goal would be to raise the bar for family medicine residency programs nationally so as to push each other to "become better than we once were." A student, resident, faculty member, or hospital administrator could use this information as "apples to apples" data to compare programs locally, regionally, and nationally. The ACGME ADS system provides measures of certain items but only in national aggregate form along with private individual program data to compare. A dashboard made available to the family medicine program director community, with more useful global measures of program health and quality, would allow every program to see if they have a smooth running vehicle that is producing graduates whose quality matches national benchmarks. Perhaps they will see that they may have a "clunker" that needs some serious work to meet standards expected by our constituents.

As a first step to embark down this road, the AFMRD board has formed a task force of past and present board members that will explore the issue and compile a list of benchmarks and quality measures. Putting these into practice and in a usable format and location will be the ultimate step. Getting buy-in from the residency community will be the key and we hope that all programs will see the benefits in this collaborative effort. Safe driving!

Grant Hoekzema, MD;

Stoney Abercrombie, MD, Sandra Carr, MDi Joseph W. Gravel Jr, MDi Karen L. Hall, MDi Stanley Kozakowski, MD, Michael F. Mazzone, MDi Todd Shaffer, MD, Martin Wieschbaus, MD 